\title{
Dynamics of Intraband and Interband Auger Processes in Colloidal Core-Shell Quantum Dots
}

\author{
Freddy T. Rabouw, ${ }^{1}$ Roman Vaxenburg, ${ }^{2}$ Artem A. Bakulin, ${ }^{3,4}$ Relinde J. A. van Dijk-Moes, ${ }^{1}$ Huib J. Bakker, ${ }^{4}$ \\ Anna Rodina, ${ }^{5}$ Efrat Lifshitz, ${ }^{2}$ Alexander L. Efros, ${ }^{6}$ A. Femius Koenderink, ${ }^{4}$ and Daniël Vanmaekelbergh ${ }^{1}$ \\ ${ }^{1}$ Condensed Matter and Interfaces, Debye Institute for Nanomaterials Science, Princetonplein 1, 3584 CC Utrecht, The Netherlands \\ ${ }^{2}$ Technion - Israel Institute of Technology, Haifa 32000, Israel \\ ${ }^{3}$ Cavendish Laboratory, University of Cambridge, JJ Thomson Ave, Cambridge CB3 OHE, UK \\ ${ }^{4}$ Center for Nanophotonics, FOM Institute AMOLF, Science Park 104, 1098 XG Amsterdam, The Netherlands \\ ${ }^{5}$ Ioffe Physical-Technical Institute, Russian Academy of Sciences, 194021 St. Petersburg, Russia \\ ${ }^{6}$ Naval Research Laboratory, Washington DC 20375, USA
}

\begin{abstract}
Conventional colloidal quantum dots (QDs) suffer from rapid energy losses by nonradiative (Auger) processes, leading to sub-ns lifetimes in all excited states but the lowest-energy single exciton. Suppression of interband Auger decay, such as biexciton Auger recombination, has been achieved with the design of heterostructured core/shell QDs. Auger-like processes are also believed to be responsible for rapid intraband hotelectron cooling in QDs. However, the simultaneous effect of shell growth on interband Auger recombination and intraband hot-electron cooling has not been addressed. Here we investigate how the growth of a CdS shell affects these two relaxation processes in $\mathrm{CdSe} / \mathrm{CdS}$ core-shell QDs. Using a combination of ultra-fast pumppush-probe spectroscopy on the QD ensemble and analysis of the photon statistics from single QDs, we find that Auger losses in the biexciton state are suppressed with increasing shell thickness, while hot-electron cooling remains unaffected. Calculations conducted within an eight-band $\mathbf{k} \cdot \mathbf{p}$ model confirm the experimental dependence of the biexciton Auger decay on the shell thickness, and provide insights into the factors determining the cooling rate of hot carriers.
\end{abstract}

\section{INTRODUCTION}

Nonradiative Auger processes, in which one charge carrier relaxes through energy transfer to another, are undesired for many applications of luminescent colloidal quantum dots (QDs). For example, under strong optical or electrical excitation QDs can become (intermittently) charged, after which Auger recombination quenches the luminescence [1-3]. The result can be a lower ensemble- and time-averaged light emission in QD LEDs [4] or spectral conversion layers [5], and dark periods in the emission from a single QD used as biolabel [6]. Auger decay of the doubly excited state of QDs (i.e. the biexciton state or $\mathrm{X}_{2}$ ) results in reduced gain in QD lasers [79]. Furthermore, an Auger-like process has been proposed to be responsible for rapid sub-ps hot-electron intraband cooling, during which the hot-electron energy is tranfered to the hole by Coulomb interaction $[10,11]$. Such fast hot-electron cooling complicates the use of QDs for exciting new technologies such as intraband infrared photodetection [12] and hot-electron solar cells [13].

Although quantitative prediction of Auger recombination rates is a difficult task, the qualitative picture is that the fast Auger rates in nanocrystal QDs are due to (1) strong electronhole wave function overlap, (2) enhancement of electron-hole Coulomb interactions by spatial and dielectric confinement [14], and (3) high and abrupt barriers in the carrier confinement potentials that result in effective breaking of the momentum conservation rule [15]. Progress has been made to suppress Auger processes in colloidal QDs, with the synthesis of heterostructures $[16,17]$. Auger decay rates of charged excitons and biexcitons can be reduced by more than factor 100 [18-20] by growing a shell into which one of the charge carriers delocalizes while the other remains confined in the QD core, or by the (intentional) formation of a compositional gradient at the core-shell interface producing soft confining potential. The interfacial alloying approach was also shown to suppress the Auger rate in quantum well LEDs [21]. Also QDs with a type-II structure, where the lowest-energy electron and hole states are spatially separated, show slow Auger decay of biexcitons [22]. In parallel, suppression of hot-electron cooling has been reported, by spatially separating electron and hole [23, 24]. In engineered type-II quantum dots, reduced electron-hole coupling was proposed to lead to an inhibition of hot-electron Auger cooling, after which coupling to vibrations of surface ligands was the next fastest cooling pathway [24]. Using this concept, slow cooling rates of $1 \mathrm{~ns}^{-1}$ [24] were reported, more than three orders of magnitude slower than the $>1 \mathrm{ps}^{-1}$ in conventional QDs [11, 25-27].

Despite the experimental successes to suppress the nonradiative (Auger) decay of charged excitons, biexcitons, and hot-carrier states, the underlying physics is not fully understood. For example, while the ensemble averaged Auger recombination rates show clear monotonic trends with QD diameter $[28,29]$, surprisingly wide variations of the Auger rate are observed in nominally identical individual QDs [30-33]. Indeed, the Auger rate was theoretically predicted to be strongly dependent on the exact geometry of individual QDs [34, 35]. Moreover, while fast hot-electron cooling is often explained in terms of electron-hole Auger coupling, this assignment is not unambiguous and the cooling pathways in QDs are still under investigation [36]. The intraband phonon-assisted Auger-like cooling of electrons is expected to be inhibited with decreasing QD size due to decreasing availability of energy-conserving transitions. However, an opposite trend was observed in experiments $[25,26]$. Moreover, intraband hole relaxation, which is the concluding step of Auger-like electron cooling, showed 
striking independence on the QD size [37], thereby suggesting additional efficient energy-conserving relaxation pathways acting alongside Auger-like cooling.

Here we compare, experimentally and theoretically, how the growth of a CdS shell on $3.8 \mathrm{~nm} \mathrm{CdSe} \mathrm{QDs} \mathrm{affects} \mathrm{the} \mathrm{rates} \mathrm{of}$ biexciton Auger recombination and $1 \mathrm{P}_{\mathrm{e}}-1 \mathrm{~S}_{\mathrm{e}}$ hot-electron cooling (Fig. 1). Using a combination of transient absorption (TA) and single-QD photoluminescence measurements we find that Auger decay of the biexciton $\left(\mathrm{X}_{2}\right.$; Fig. 1a) state in QDs with a thick 18 monolayer shell is suppressed by more than an order of magnitude compared to QDs with a thin 1-2 monolayer shell. Similar suppression of $\mathrm{X}_{2}$ Auger recombination in thick shell QDs follows from theoretical calculations using an eightband $\mathbf{k} \cdot \mathbf{p}$ model, if we take into account the size distribution of the QD ensemble. On the same batches of QDs we perform ultrafast pump-push-probe experiments to investigate hot electron cooling (Fig. 1b) [10]. While $\mathrm{X}_{2}$ Auger recombination is suppressed by the growth of a $\mathrm{CdS}$ shell, the cooling of hot electrons is unaffected. This finding is at odds with the standard model of hot-electron cooling by Auger coupling with the hole, because in this model the cooling rate would be reduced for increasing shell thickness as the hot $1 \mathrm{P}_{\mathrm{e}}$ electron delocalizes. We suggest that the effect of delocalization could be compensated by a second counteracting effect, and discuss which effects may be involved. Furthermore, the low density of hole states in the valence band obtained from calculations suggests that rapid carrier cooling in QDs with small cores cannot be explained in terms of the simple picture of electron-hole Auger coupling alone. Such coupling must be strongly phonon-assisted or even be complemented with alternative cooling pathways.

\section{SLOW-DOWN OF BIEXCITON AUGER DECAY}

We examine three batches of CdSe/CdS core/shell QDs. They all have the same CdSe cores with a diameter of 3.8 $\mathrm{nm}$, but with a different number of CdS layers grown with the SILAR method (see Methods). There are two batches of thinshell QDs, with 1 monolayer of CdS and a total diameter of 4.8 $( \pm 0.5) \mathrm{nm}$, and with 2 monolayers and a total diameter of 6.0 $( \pm 0.5) \mathrm{nm}$. A batch of thick-shell QDs has 18 monolayers of $\mathrm{CdS}$, and slightly anisotropic shape of $24( \pm 2) \mathrm{nm}$ by $16( \pm$ 2) nm. Representative transmission electron microscope images, ensemble emission spectra and photoluminescence decay curves are shown in the Supplementary Figure S1.

Figure 2 shows the TA signal in the infrared (IR; recorded at $1700 \mathrm{~cm}^{-1}=0.21 \mathrm{eV}$ ) of the three QD batches, upon pumping at $400 \mathrm{~nm}$. Pump absorption creates conduction band electrons and valence band holes, resulting in an induced intraband absorption band in the IR. The probe energy of $0.21 \mathrm{eV}$ corresponds roughly to the single-particle $1 \mathrm{~S}_{\mathrm{e}} \rightarrow 1 \mathrm{P}_{\mathrm{e}}$ transition in the conduction band [39] (see also Supplementary Fig. S1). This transition has much larger absorption cross-section than any intraband transition in the valence band from the $1 S_{3 / 2}$ ground state of the hole [40]. Hence, the magnitude of the TA signal reflects the concentration of excited electrons in the $1 \mathrm{~S}_{\mathrm{e}}$ state. At low excitation fluences (blue and green in Fig. 2) the TA decays slowly, with fitted time constants of longer than a nanosecond for all three QD batches (Fig. 2a,b,c). This slow component is due to radiative decay of single excitons, i.e. relaxation of conduction band electrons by radiative recombination with valence band holes.

At higher excitation fluences (yellow, orange and red in Fig. 2) there is a significant probability that within a single laser pulse a QD absorbs two photons, resulting in biexciton $\left(\mathrm{X}_{2}\right)$ or higher multi-exciton states. These decay much more quickly than the single exciton state, because (1) radiative decay of multi-exciton states is faster than single-exciton decay [38] and (2) they can additionally decay via non-radiative Auger recombination. The fast component in the TA signal of thin-shell QDs (Figs. 2a,b) at high fluences (yellow, orange, red) is due to these fast processes. We fit triple-exponential functions to the data (see figure caption for details), and obtain the $\mathrm{X}_{2}$ lifetimes. We find a $\mathrm{X}_{2}$ lifetime of $90 \mathrm{ps}$ for the 1-monolayer batch (Fig. 2a) and 96 ps for the 2-monolayers batch (Fig. 2b). Subtracting the estimated radiative decay rates of the $\mathrm{X}_{2}$ state (see Supplementary Figure S1), we obtain $\mathrm{X}_{2}$ Auger lifetimes of 92 ps and 98 ps, respectively. These are slightly longer than typical biexciton Auger lifetimes of 3040 ps of bare CdSe QDs with the size of our cores (1.9 nm radius) $[41,42]$.

In sharp contrast to the thin-shell QDs, the measurements on the thick-shell QDs at high excitation fluences (yellow and red in Fig. 2c) do not show a fast component. We see nevertheless that the decay becomes faster. This indicates that the creation of biexcitons does occur, but that $\mathrm{X}_{2}$ Auger recombination in these QDs is slower than the 500 ps time scale of our TA experiments. A single-exponential function yields a good fit to the data even at the highest excitation fluence of $100 \mu \mathrm{J} \mathrm{cm}^{-2}$ (red in Fig. 2c), yielding a time constant of longer than $1 \mathrm{~ns}$. The slow-down of $\mathrm{X}_{2}$ Auger recombination with the growth of a $\mathrm{CdS}$ shell (from $<100 \mathrm{ps}$ to $>1 \mathrm{~ns}$ ) is consistent with previous investigations [18]. From an estimate of the radiative decay rates of $\mathrm{X}_{2}$ (see Supplementary Figure S1) the obtained $\mathrm{X}_{2}$ lifetimes correspond to an average quantum efficiency of the $\mathrm{X}_{2} \rightarrow \mathrm{X}$ transition of $2 \%$ for the thin-shell QDs, and $>28 \%$ for the thick-shell QDs.

To confirm the observed suppression of Auger recombination and quantify the slow $\mathrm{X}_{2}$ Auger decay rate in single thickshell QDs, we performed time-correlated single photon counting (TCSPC) experiments on single QDs. Figures 3a,b present the two-photon correlation function $g^{(2)}$ of the emission from (a) a single thin-shell QD (2 monolayers), and (b) a single thick-shell QD (18 monolayers). At low excitation fluences the zero-delay peak in such plots is proportional to the quantum yield ratio between the $\mathrm{X}_{2} \rightarrow \mathrm{X}$ and the $\mathrm{X} \rightarrow 0$ transitions [43] (where 0 denotes the ground state of the QD). Fast $\mathrm{X}_{2}$ Auger recombination in the thin-shell QD leads to a $\mathrm{X}_{2}$ quantum yield of nearly zero (see above). Indeed, there is no zerodelay peak visible over the noise in the $g^{(2)}$-plot (Fig. 3a). The thick-shell QD, on the other hand, shows a pronounced zero- 
a

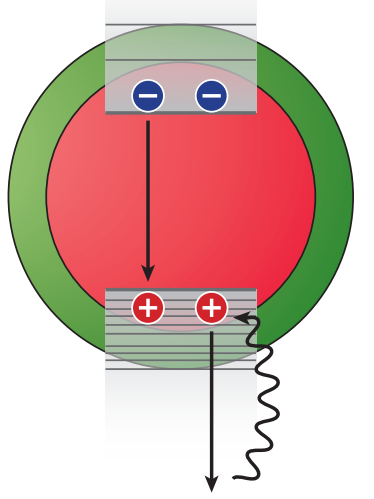

b hot electron cooling

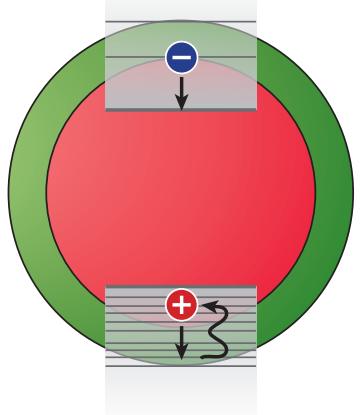

FIG. 1. (a) Biexciton Auger decay is dominated by the 'positive trion pathway' $[29,38]$, in which the recombination energy of one electronhole pair is transferred to the remaining hole (straight arrows). The excited hole can relax back to the top of valence band by phonon emission (wavy arrow). (b) Hot electron Auger cooling is the relaxation of an electron to the bottom of the conduction band through energy transfer to a valence band hole (straight arrows). Subsequently, the hole rapidly cools down to the top of the valence band by emission of phonons (wavy arrow).
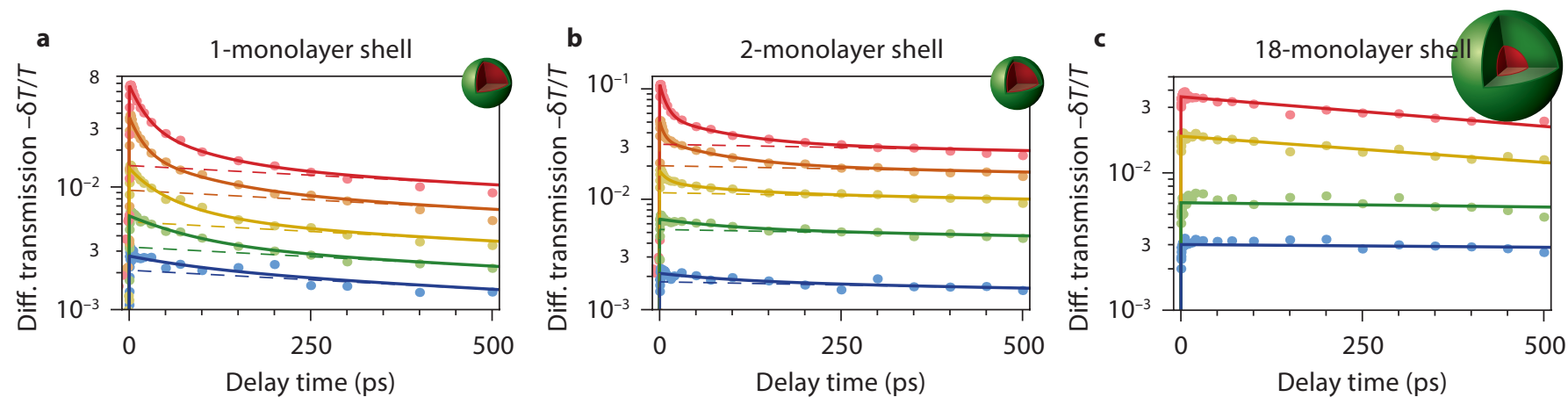

FIG. 2. (a) Induced transient mid-IR absorption at the $1 \mathrm{~S}_{\mathrm{e}} \rightarrow \mathrm{P}_{\mathrm{e}}$ transition $\left(1700 \mathrm{~cm}^{-1}\right)$ in thin-shell QDs with 1 monolayer of CdS, at fluences of the $400 \mathrm{~nm}$ pump of 5 (blue), 10 (green), 20 (yellow), 50 (orange), and 100 (red) $\mu \mathrm{J} \mathrm{cm}{ }^{-2}$. (b) Transient absorption in thin-shell QDs with 2 monolayers of CdS, at pump fluences of 2.5 (blue), 9 (green), 25 (yellow), 50 (orange), and 100 (red) $\mu \mathrm{J} \mathrm{cm}{ }^{-2}$. (c) Transient absorption in

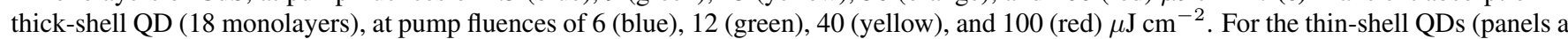
and $b$ ) the dashed lines are single-exponential fits to the data points between 200 and $500 \mathrm{ps}$ of the lowest-fluence measurement (blue symbols), yielding (a) $\tau_{1}=1.4 \mathrm{~ns}$, and (b) and $\tau_{1}=3.8 \mathrm{~ns}$. The fixed value of $\tau_{1}$ and two additional time constants are used in tri-exponential fits to the full curves (solid lines). We interpret the faster time constants of (a) $\tau_{2}=90 \pm 12 \mathrm{~ns}$ (average \pm standard deviation over fits to the different excitation fluences) and $\tau_{3}=20 \pm 5 \mathrm{~ns}$, and (b) $\tau_{2}=96 \pm 9 \mathrm{~ns}$ and $\tau_{3}=7 \pm 2 \mathrm{~ns}$ as due to decay of biexcitons $\left(\tau_{2}\right)$ and multi-excitons $\left(\tau_{3}\right)$. We fit the data of the thick-shell QDs (c) to single-exponentials, yielding time constants between $11 \mathrm{~ns}$ (blue) and $1 \mathrm{~ns}$ (red).

delay peak (Fig. 3b). From its amplitude, and assuming that $\mathrm{X}$ (see Supplementary Figure S2) has a near-unity quantum yield, we estimate that the $\mathrm{X}_{2}$ quantum yield in this particular QD is $34 \%$.

It is possible to directly quantify the $\mathrm{X}_{2}$ lifetime from a TCSPC experiment. To this end, one must construct the twophoton correlation function $g^{(2)}$ of the emission from a single QD after first rejecting all photon counts that come within a blind period of variable length $\Delta$ after the laser pulse $[44,45]$. The principle is illustrated in Fig. 3c. Laser pulses are given at regular time intervals (green bars), while from time to time an emitted photon is detected (red bars) on either detector. Sometimes a laser pulse is followed by two photon detection events, one on both detectors (highlighted in blue). These events are due to $X_{2}$ cascade emission. Since the first step of the $\mathrm{X}_{2}$ cascade is fast (typically within a ns), by applying a short blind period $\Delta$ after the laser pulses (gray shaded areas) one rejects coincidence counts from $\mathrm{X}_{2}$ cascade emission more strongly than random coincidences from consecutive $\mathrm{X}$ emissions. Consequently, the integrated area of the zero-delay peak in the $g^{(2)}$-plot decreases more strongly with increasing blind time than those of the side peaks.

Fig. 3d shows the two-photon correlation functions $g^{(2)}$ (of the same single QD as examined in Fig. 3b) constructed after applying different blind times $\Delta$ of $0 \mathrm{~ns}$ (red), $5 \mathrm{~ns}$ (yellow), 10 ns (green), and $20 \mathrm{~ns}$ (blue). As expected, the area of the zerodelay peak decreases more rapidly with increasing blind time than for the side peaks. In Fig. 3e we plot how the peaks decay 
a

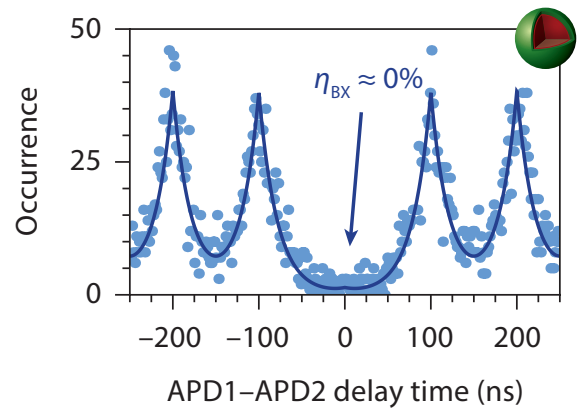

c

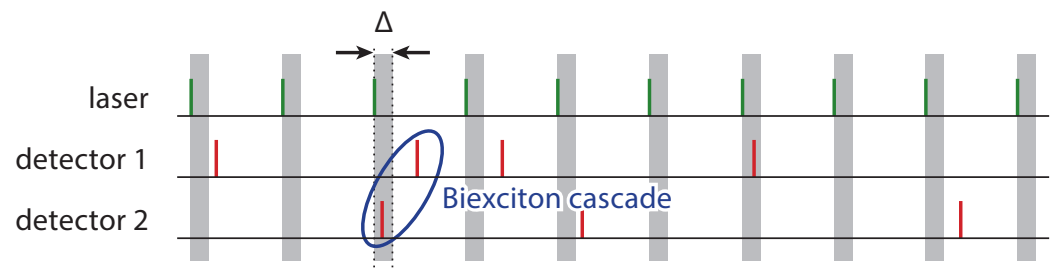

Experiment time $(T)$

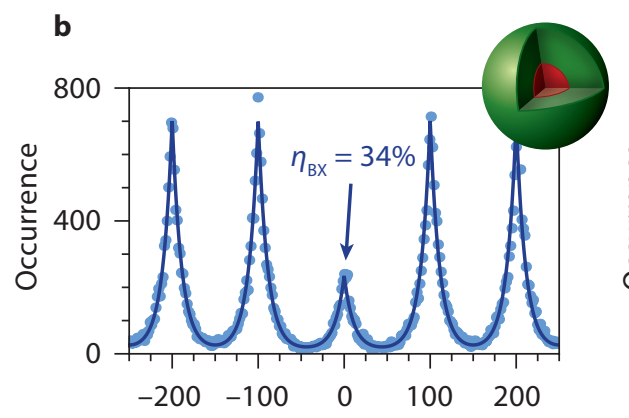

APD1-APD2 delay time (ns) d

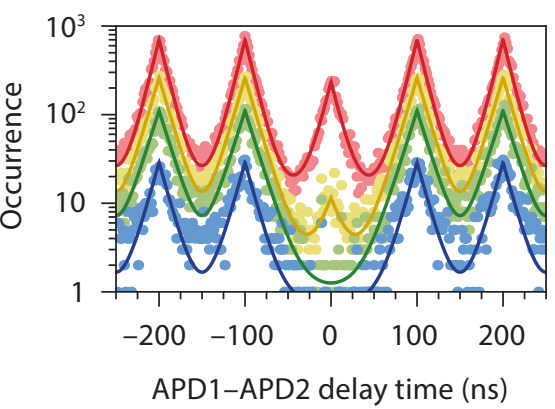

e

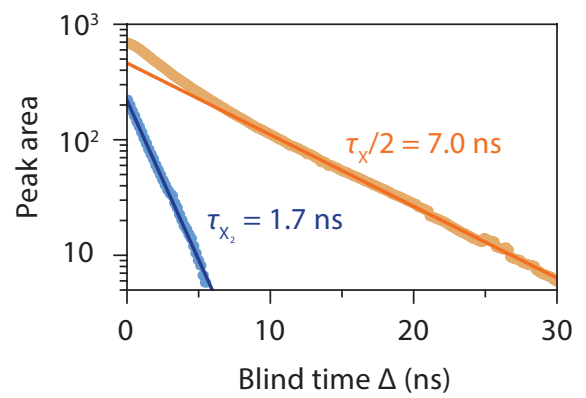

FIG. 3. (a,b) The two-photon correlation function $g^{(2)}$ of the emission from (a) a single thin-shell QD (2 monolayers), and (b) a single thickshell QD (18 monolayers). From the relative peak area of the zero-delay peak [43] we estimate $\mathrm{X}_{2}$ quantum efficiencies of near 0\% in (a) and $34 \%$ in (b). (c) Schematic of the signals recorded during a time-correlated single photon counting experiment. Laser pulses (green bars) come at regular intervals. The two detectors, both aligned on the same single QD, sometimes detect a photon (red bars). Two consecutive photon detection events after a single laser pulse must originate from a biexciton cascade (highlighted in blue). For our analysis we discard detection events within a short variable blind time $\Delta$ (gray shaded area) after the laser pulse. (d) The $g^{(2)}$-plot of a single thick-shell QD constructed with blind times of $\Delta=0 \mathrm{~ns}$ (red), $5 \mathrm{~ns}$ (yellow), $10 \mathrm{~ns}$ (green), and $20 \mathrm{~ns}$ (blue). Solid lines are fits to a regular array of exponentially decaying peaks. (e) The peak area of the zero-delay peak (blue) and the side peaks (orange) as a function of blind time $\Delta$. The zero-delay peak area decays exponentially with a time constant equal to $\mathrm{X}_{2}$ lifetime, for which we fit $\tau_{\mathrm{X}_{2}}=1.7 \mathrm{~ns}$.

with increasing $\Delta$. The decay of the side peaks (orange data points) contains a fast contribution from $\mathrm{X}_{2}$ emission, while the slow component should decay with a time constant equal to half the $\mathrm{X}$ lifetime (see the Supplementary Information for a derivation). The orange solid line is a fit from which we extract an X lifetime of $14.1 \mathrm{~ns}$, consistent with the $16.3 \mathrm{~ns}$ obtained from the PL decay curve of this QD (see SI Fig. S2). The zero-delay peak (blue data points) is mostly due to $\mathrm{X}_{2}$ cascades. It decays with the $\mathrm{X}_{2}$ lifetime, for which we fit 1.7 ns (solid line). From this analysis and the $\mathrm{X}$ lifetime of 16.3 ns, we estimate an Auger lifetime of $2.8 \mathrm{~ns}$. In other single QDs from this batch we consistently find $\mathrm{X}_{2}$ Auger lifetimes of the order of a ns: $1.4 \pm 0.6 \mathrm{~ns}$ over 11 QDs.

The above results clearly reveal the trend that in $\mathrm{CdSe} / \mathrm{CdS}$ core/shell QDs the $\mathrm{X}_{2}$ Auger rate slows down with the growth of a CdS shell. The $\mathrm{X}_{2}$ Auger rate in the thick-shell sample is slower than in the thin-shell samples by a factor 15 , and slower than in core-only QDs by a factor 30-40 [41, 42]. In Fig. 4 we compare this suppression of the Auger recombination observed in experiment with calculations of the $\mathrm{X}_{2}$ Auger rate performed using an 8 -band $\mathbf{k} \cdot \mathbf{p}$ model (see Methods). In the calculations, as in the experiment, we consider $\mathrm{CdSe} / \mathrm{CdS}$ QDs with fixed core diameter of $3.8 \mathrm{~nm}$ and variable shell thickness between 0 and 18 monolayers. We assume that after the Auger recombination event, the excited charge carrier (either an electron or a hole) resides in the continuous spectrum above the outer barrier of the QD. CdSe/CdS heteronanocrystals have a type- $\mathrm{I} \frac{1}{2}$ band alignment [46] with holes strongly confined to the core region. The magnitude of the (small) conduction band offset at the $\mathrm{CdSe} / \mathrm{CdS}$ interface is however not well established [18]. Values in the range between 0 and 300 $\mathrm{meV}$ are typically reported [47-49], and in our calculations we consider the offset energy as a variable parameter within that range. The rest of the material parameters used in the calculations are from Refs. $50(\mathrm{CdSe})$ and $51(\mathrm{CdS})$.

Figure 4 shows the calculated $\mathrm{X}_{2}$ Auger rates as a function of shell thickness in individual QDs (blue curves), and averaged over a QD ensemble with 10\% size dispersion (red curves) for three conduction band offsets $\Delta E_{\mathrm{cb}}=0,100,200$ $\mathrm{meV}$. The experimental data for thin- and thick-shell QDs stud- 

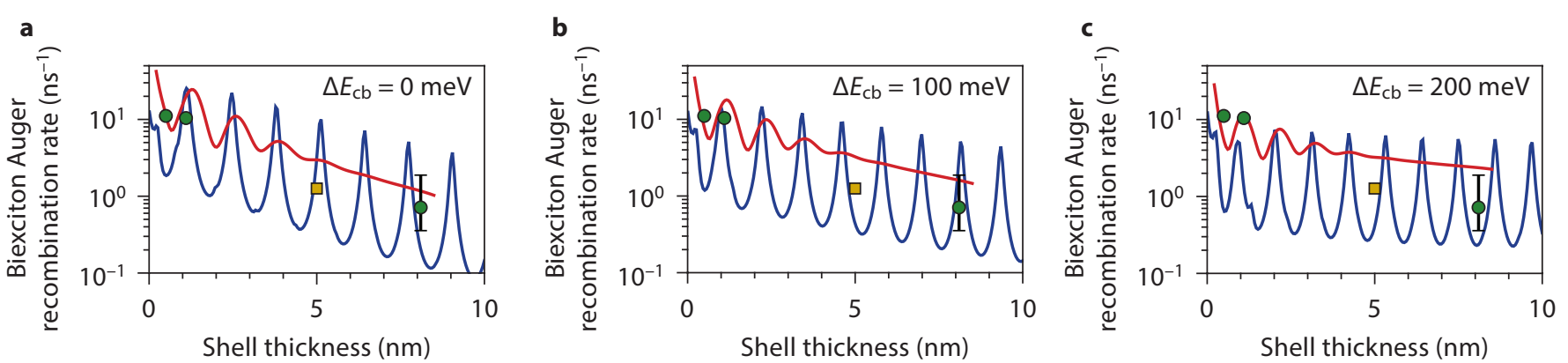

FIG. 4. Calculated (lines) and experimental (symbols) $\mathrm{X}_{2}$ Auger decay rates as a function of shell thickness in CdSe/CdS core-shell QDs with $3.8 \mathrm{~nm}$ core diameter, for a conduction band offset of (a) $\Delta E_{\mathrm{cb}}=0$, (b) $\Delta E_{\mathrm{cb}}=100 \mathrm{meV}$, or (c) $\Delta E_{\mathrm{cb}}=200 \mathrm{meV}$. The blue and red lines are the calculated Auger rates in individual QDs and averaged over a QD ensemble with 10\% size distribution (in the size of both core and shell, uncorrelated), respectively. The green circles are the experimental data for QDs investigated in this work, having 1-, 2-, and 18-monolayer shells (with Auger lifetimes of $\tau_{\mathrm{A}}=92 \mathrm{ps}, 98 \mathrm{ps}$, and $1.4 \mathrm{~ns}$, respectively). The bars on the data point for the 18-monolayer QDs depict the full variation of Auger lifetimes measured on 11 different single QD. The yellow square represents a comparable QD sample having $2.0 \mathrm{~nm}$ core radius, $5.0 \mathrm{~nm}$ shell thickness, and $\tau_{\mathrm{A}}=0.75 \mathrm{~ns}$ from Ref. 19 .

ied here are shown by green symbols. The yellow symbol represents a QD sample of intermediate shell thickness from Ref. 19. The calculations are in reasonable agreement with the experiment, both in terms of the absolute values of the $\mathrm{X}_{2}$ Auger recombination rates, and on the overall trend of decreasing rate with increasing shell thickness. One can see in Fig. 4 that this trend is more pronounced for smaller $\Delta E_{\mathrm{cb}}$. This is because with decreasing conduction band offset the electron can delocalize more strongly into the shell, thereby reducing the electron-hole overlap and Coulomb interaction. The best agreement between calculation and experiment is obtained if we assume that $\Delta E_{\mathrm{cb}}=0$. Further, the Auger rate in individual QDs decreases with increasing shell thickness in a nonmonotonic way, with oscillations of approximately an order of magnitude wide. These oscillations explain why the $\mathrm{X}_{2}$ Auger rate measured on single QDs from one batch with slight variations in geometry varies widely (in our case, using the method of Fig. 3, between 0.5 ns and 2.8 ns; depicted in Fig. 4) [3033]. However, the oscillations average out in ensemble-based experiments (Fig. 2), as depicted by the red line in Fig. 4. In the calculation of the ensemble-averaged Auger rate (red line in Fig. 4), uncorrelated size dispersions of $10 \%$ in both core size and shell thickness were assumed. The blue line depicts the calculations for a specific core diameter of $3.8 \mathrm{~nm}$ only.

It should be noted that the agreement between experiment and model in Fig. 4 can possibly be improved. Currently, the model assumes an abrupt $\mathrm{CdSe} / \mathrm{CdS}$ interface between the core and the shell of the QDs. It has been demonstrated that Auger rates are affected if the interface is "smoothened" by alloying [18-20]. In our samples of core-shell QDs unintentional alloying might occur because the shell in grown layer-by-layer over a duration of hours at high temperature [52]. The extent of smoothening of the interface could be introduced in the model as an unknown parameter. However, since there is already a reasonable consistency between the experiment and the model with an abrupt interface, we conclude that in the current experiment interfacial alloying does not have a pronounced ef- fect. Therefore, the main mechanism responsible for the reduced Auger rates with increasing shell thickness is a reduction of the spatial overlap between ground state electron and hole wavefunctions, and loss of high-momentum components in the delocalized electron wavefunction. Another point on which our model could be fine-tuned, is the dielectric screening. We have currently used the dielectric constant of the bulk material to describe the dielectric screening of the Coulomb interaction in QDs of all sizes. In small QDs, however, dielectric screening can be reduced compared to the bulk material [53]. In Supplementary Figure S3 we show the effect of introducing the reduced dielectric screening as an input parameter. In the case of thick shells, the model of the Auger recombination can be also improved by taking into account the weak adiabatic Coulomb potential imposed on the electron by the strongly confined hole [49, 54].

\section{HOT ELECTRON COOLING IN THE CONDUCTION BAND}

Next, we use ultrafast pump-push-probe experiments [40, 56] to determine the rate of hot electron cooling in QDs with different shell thickness. The experimental method is illustrated in Figure 5a. A pump pulse of $400 \mathrm{~nm}(=3.1 \mathrm{eV}$; blue arrow in Fig. 5a) creates excited electrons in the conduction band. A probe pulse records the induced absorption at the intra-conduction band $1 \mathrm{~S}_{\mathrm{e}} \rightarrow 1 \mathrm{P}_{\mathrm{e}}$ transition at $1700 \mathrm{~cm}^{-1}$ (= $0.21 \mathrm{eV}$; dashed red arrow in Figs. 5a). What makes our pumppush-probe experiment different from normal pump-probe, is that a third push pulse of $1850 \mathrm{~cm}^{-1}(=0.22 \mathrm{eV}$; red arrow in Fig. 5a) can excite an electron from the lowest-energy $1 \mathrm{~S}_{\mathrm{e}}$ level in the conduction band, to the higher lying $1 \mathrm{P}_{\mathrm{e}}$ level. This push leads to a partial bleach of the $1 \mathrm{~S}_{\mathrm{e}} \rightarrow 1 \mathrm{P}_{\mathrm{e}}$ absorption induced by the first pump pulse and measured by the probe pulse. The evolution of the transient absorption signal is schematically depicted in Figure 5b, with the timing of the pump and the push pulse indicated. We are particularly interested in the 
recovery of the bleach signal (highlighted in green in Fig. 5b), as it reveals the rate at which hot $1 \mathrm{P}_{\mathrm{e}}$ electrons cool down (labeled $\mathrm{C}$ in Fig. 5a) to the $1 \mathrm{~S}_{\mathrm{e}}$ level.

Figures $5 \mathrm{c}$,d,e show the recovery of the $1 \mathrm{~S}_{\mathrm{e}} \rightarrow 1 \mathrm{P}_{\mathrm{e}}$ absorption as hot electrons cool down after the push pulse, for the three QD batches. These plots are a zoom-in of the total transient absorption trace on the region highlighted in green in the schematic graph of Fig. 5b. For all batches the absorption returns to the value of before the push pulse on a sub-ps timescale, evidencing fast and efficient cooling of generated $1 \mathrm{P}_{\mathrm{e}}$ electrons back to the $1 \mathrm{~S}_{\mathrm{e}}$ level. We fit the recovery of the absorption signal to single-exponential decay convoluted with a Gaussian instrument response function of $70 \mathrm{fs}$, and obtain fitted hot electron lifetimes of $800 \mathrm{fs}$ for the thin-shell QDs with 1 monolayer shell (Fig. 5c), 736 fs for the thin-shell QDs with 2 monolayers shell (Fig. 5d), and 510 fs for the thick-shell QDs (18 monolayers; Fig. 5e). These values are consistent with previously reported values for core-only QDs: $220 \mathrm{fs}$ determined from two-photon photoemission spectroscopy [27], 900-1200 fs from pump-push-probe experiments [23], or 100$600 \mathrm{fs}$ from TA experiments in the visible [26]. The results of Figure 5 demonstrate that the growth of a $\mathrm{CdS}$ shell does not suppress hot electron cooling in CdSe QDs. Interestingly, we also see that the presence of additional charge carriers does not affect hot-electron cooling. The red data points in Fig. 5c depict the cooling in the biexciton regime (i.e. at high pump fluence), which is roughly equally fast as in the single-exciton regime (blue data points).

Fast sub-ps hot electron cooling has been proposed to be an Auger process, where the hot electron transfers the relaxation energy to a valence band hole [10, 23, 26]. This Auger-type cooling is necessary because of the so-called phonon bottle neck in colloidal QDs: 'normal' cooling through the emission of phonons is not possible because there are no phonon modes of sufficiently high energy to bridge the separation between the conduction band levels [10]. Instead, rapid hot-electron cooling is enabled by Auger coupling with valence band holes, which is especially efficient because of spatial confinement of electrons and holes. In fact, electrons and holes are so tightly co-localized in colloidal QDs that in the smallest QDs the rate of Auger-type cooling can exceed the rate of cooling by emission of phonons in the corresponding bulk material [26]. Using time-resolved terahertz spectroscopy, Hendry et al. [11] have found evidence that in CdSe QDs there is indeed energy transfer from the hot electron to the hole. Pandey and GuyotSionnest [24] rendered Auger-type cooling impossible by spatially separating electron and hole in type-II QDs with surface hole traps. In these specially designed QDs the electron cooling was reported to be as slow as a ns, and in this regime to be dominated by coupling to vibrations of the organic surface ligands. The equal cooling rates in the single-exciton and biexciton regimes (Fig. 5c) are consistent with Auger cooling if we consider statistical scaling: the biexciton state provides twice as many hole acceptors ( 2 rather than 1$)$, but twice as few final states for the hot electron (1 rather than 2).

\section{DISCUSSION}

Table I summarizes the values found for the $\mathrm{X}_{2}$ Auger lifetimes and the cooling rates in thin-shell and thick-shell QDs. Although hot-electron cooling is often attributed to an Augerlike process, in our experiments it is not affected by the growth of a CdS shell in the same way as $\mathrm{X}_{2}$ Auger recombination. Intuitively one would expect that also hot-electron Auger cooling were suppressed by shell growth. Indeed, the hot $1 \mathrm{P}_{\mathrm{e}}$ state in the conduction band strongly delocalizes in the $\mathrm{CdS}$ shell (Fig. 6a), so that the Coulomb interaction with the hole as well as the overlap with the final $1 \mathrm{~S}_{\mathrm{e}}$ electron state would reduce. This simple picture of hot-electron Auger coupling is not fully consistent with the experiment (Fig. 5). An important difference between $\mathrm{X}_{2}$ Auger recombination and hot-electron Auger cooling is that in the former case the hole is excited to basically a continuum of states, while it is excited to one of the discrete quantum confined states in the latter case.

Fig. $6 \mathrm{~b}$ presents the calculated energy level structure of the quantum confined hole states as a function of shell thickness in $\mathrm{CdSe} / \mathrm{CdS}$ core-shell QDs with a $3.8 \mathrm{~nm}$ diameter core, and conduction band offset of $200 \mathrm{meV}$. Since holes in this type-I $\frac{1}{2}$ structure are tightly confined to the CdSe core, the hole energy levels can be calculated assuming strong confinement for all shell thicknesses. The energy levels involved in $1 \mathrm{P}_{\mathrm{e}}-1 \mathrm{~S}_{\mathrm{e}}$ hotelectron cooling are depicted as solid lines in Fig. 6b, the rest as dotted lines. Of the valence band transitions, only those from the $1 S_{3 / 2}$ hole ground state to excited states with $\mathrm{P}$ symmetry can couple to the $1 \mathrm{P}_{\mathrm{e}}-1 \mathrm{~S}_{\mathrm{e}}$ transition in the conduction band. We see that the energy levels of the hole are nearly independent of the shell, because the hole remains tightly confined in the CdSe core for all shell thicknesses. Importantly, we see that the energy level structure in the valence band contains large gaps of $>80 \mathrm{meV}(\approx 3$ longitudinal optical phonons of $\mathrm{CdSe}$ or $\mathrm{CdS}$ ). For instance, there is a surprisingly large gap of almost $100 \mathrm{meV}$ between the first $\left(1 \mathrm{P}_{3 / 2}\right)$ and second $\left(1 \mathrm{P}_{5 / 2}\right)$ excited hole states, and more gaps at higher energy.

In Fig. 6c we plot the theoretical energies for the intraband transitions involved in hot-electron Auger cooling as a function of shell thickness. The energy of the electron transition $1 \mathrm{P}_{\mathrm{e}} \rightarrow 1 \mathrm{~S}_{\mathrm{e}}$ are shown in blue, while these of the simultaneous hole transitions $1 \mathrm{~S}_{3 / 2} \rightarrow n \mathrm{P}_{j}(j=1 / 2,3 / 2,5 / 2)$ are shown in red. For the electron transition in $\mathrm{CdSe} / \mathrm{CdS}$ core-shell QDs the strong-confinement approximation holds only for thin-shell QDs, whereas for thick shells the adiabatic Coulomb potential produced by the strongly confined hole becomes important [54]. We therefore calculate the $1 \mathrm{P}_{\mathrm{e}} \rightarrow 1 \mathrm{~S}_{\mathrm{e}}$ transition energy within the strong-confinement approximation for thin shells up to $3 \mathrm{~nm}$ (blue solid line), and including the adiabatic Coulomb potential generated by the hole for thick shells $(>5 \mathrm{~nm}$ ). The intersections of the curves for electron (blue) and hole (red) in Fig. 6c indicate energyconserving Auger cooling transitions. One can see that the electron $1 \mathrm{P}_{\mathrm{e}}-1 \mathrm{~S}_{\mathrm{e}}$ transition is exactly resonant with one of the hole transitions in the valence band only for a few very spe- 
a

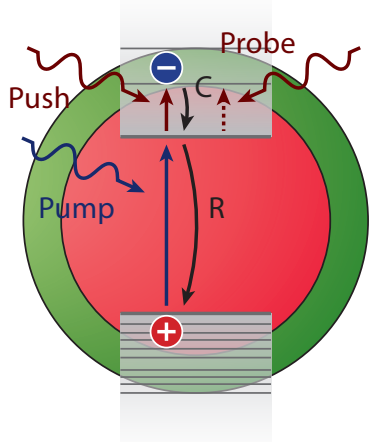

b

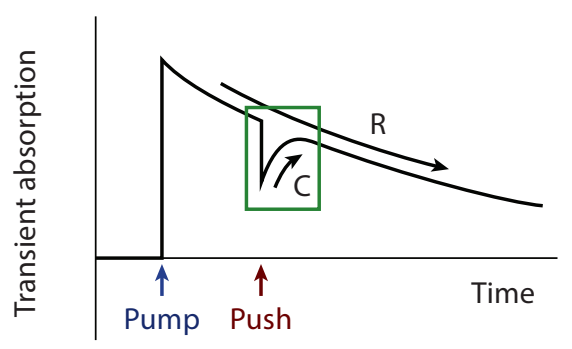

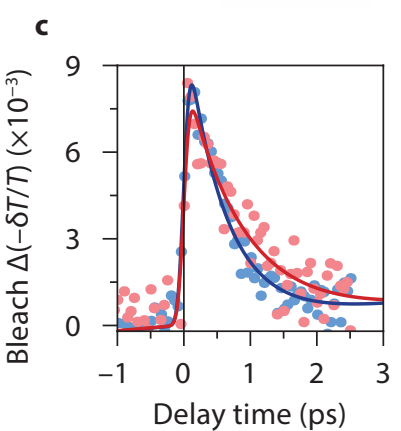
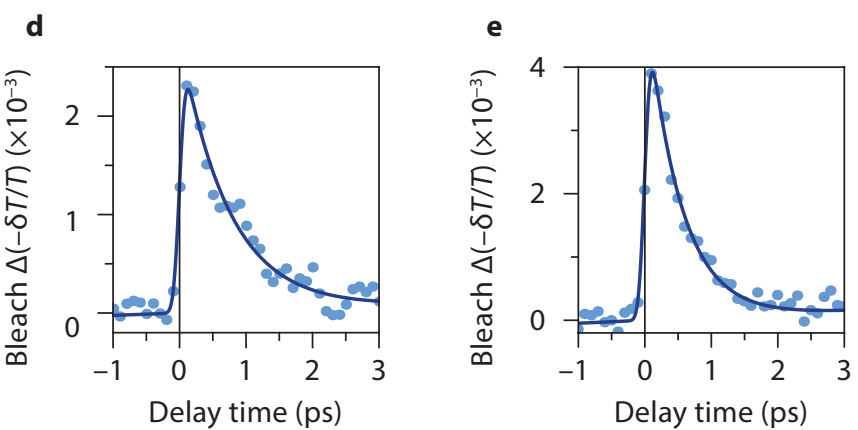

FIG. 5. (a) In a pump-push-probe experiment an electron is excited to the conduction with a pump pulse of $400 \mathrm{~nm}$ (blue arrow). After relaxation to the $1 \mathrm{~S}_{\mathrm{e}}$ level a short push pulse of $1850 \mathrm{~cm}^{-1}$ (red arrow) excites it to the $1 \mathrm{P}_{\mathrm{e}}$ level. The electron relaxes by first cooling down to the $1 \mathrm{~S}_{\mathrm{e}}$ level (labeled $\mathrm{C}$ ), and then (radiative) recombination with the valence band hole (labeled $\mathrm{R}$ ). A probe pulse at $1700 \mathrm{~cm}^{-1}$ records the absorption at the $1 \mathrm{~S}_{\mathrm{e}} \rightarrow 1 \mathrm{P}_{\mathrm{e}}$ transition, which is induced by the pump but bleached by the probe. (b) Schematic of the absorption transient at the $1 \mathrm{~S}_{\mathrm{e}} \rightarrow 1 \mathrm{P}_{\mathrm{e}}$ transition $\left(1700 \mathrm{~cm}^{-1}\right.$ ) in reaction to the pump pulse (blue arrow) inducing absorption and the push pulse (red arrow) partially bleaching it. The recovery of the signals reveals the rates of cooling $(\mathrm{C})$ and recombination (R). (c,d,e) The differential transmission transient due to $1 \mathrm{~S}_{\mathrm{e}} \rightarrow 1 \mathrm{P}_{\mathrm{e}}$ excitation by the push pulse and subsequent electron cooling for (c) thin-shell QDs with 1 monolayer shell, (d) thin-shell QDs with 2 monolayers shell, and (e) thick-shell QDs. Note that the plot is inverted compared to the cartoon in b. In (d) and (e) the pump fluence was $20 \mu \mathrm{J} \mathrm{cm}^{-2}$ and the pump-push delay $200 \mathrm{ps}$, so that at the moment of the push only single excitons exist. In panel (c) the pump-push delay is $40 \mathrm{ps}$, and the experiment was done at two pump fluences of $5 \mu \mathrm{J} \mathrm{cm}^{-2}$ (blue; only single excitons) and $200 \mu \mathrm{J} \mathrm{cm}^{-2}$ (red; also biexcitons). Solid lines are single-exponential fits, yielding in panel (c) $800 \mathrm{fs}$ (blue; low fluence) and 859 fs (red; high fluence), in panel (d) $736 \mathrm{fs}$, and in panel (e) $510 \mathrm{fs}$.

$\begin{array}{ccc}\text { CdS shell thickness (\# monolayers) } & \mathrm{X}_{2} \text { Auger lifetime } & \text { inverse cooling rate } \\ 1 & 92 \mathrm{ps} & 800 \mathrm{fs} \\ 2 & 98 \mathrm{ps} & 736 \mathrm{fs} \\ 18 & 1.4 \pm 0.6 \mathrm{~ns} & 510 \mathrm{fs}\end{array}$

TABLE I. Summary of the values found for the $\mathrm{X}_{2}$ Auger lifetimes and the cooling rates in CdSe/CdS core-shell QDs. The $\mathrm{X}_{2}$ Auger lifetimes of the thin-shell samples (1 and 2 monolayers) are fitted from transient absorption curves (Fig. 2), of the thick-shell sample (18 monolayers) from single-QD spectroscopy (Fig. 3). The cooling rates are fitted from the recovery of IR absorption in pump-push-probe experiments (Fig. 5).

cific shell sizes. Clearly, since sub-ps cooling times have been measured for many different QD geometries (see Fig. 5 and Refs. [11,25-27]), there must be a mechanism to compensate for the energy mismatch in the energy transfer from the excited $1 \mathrm{P}_{\mathrm{e}}$ electron to the ground state $1 \mathrm{~S}_{3 / 2}$ hole. Furthermore, the presence of large energy gaps in the valence band implies that while the model of Auger cooling can explain the absence of a phonon bottleneck for the electron, it would still be there for the hole.

Even if we assume strong homogeneous broadening of the excited hole states in the valence band of $\hbar / 50 \mathrm{fs}=13 \mathrm{meV}$, the large energy gaps in the valence band and large mismatches between the electron and hole intraband transitions remain. Since the maximum phonon energy in CdSe is only $26 \mathrm{meV}$, multi-phonon processes must be involved to compensate the energy mismatches $[10,11]$. Hence, if hot-electron Auger cooling is indeed operative in $\mathrm{CdSe}$ and $\mathrm{CdSe} / \mathrm{CdS}$ core/shell QDs, both steps in the process (i.e. energy transfer to the hole, and cooling of the hole) are strongly dependent on phonon assistance. Indeed, atomistic calculations by Kilina et al. [36] have indicated that multi-phonon processes are important in hot-carrier cooling. Since the cooling pathways must be 
a

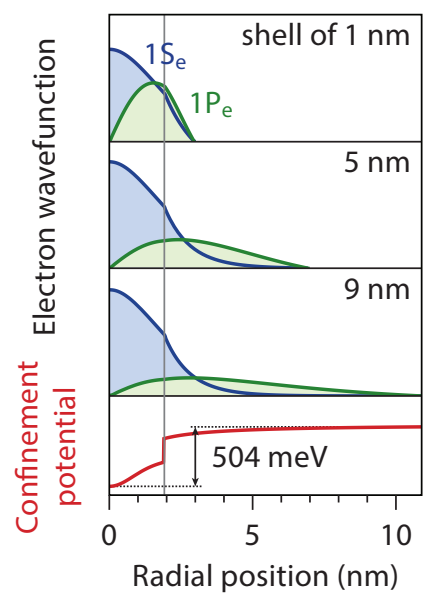

b

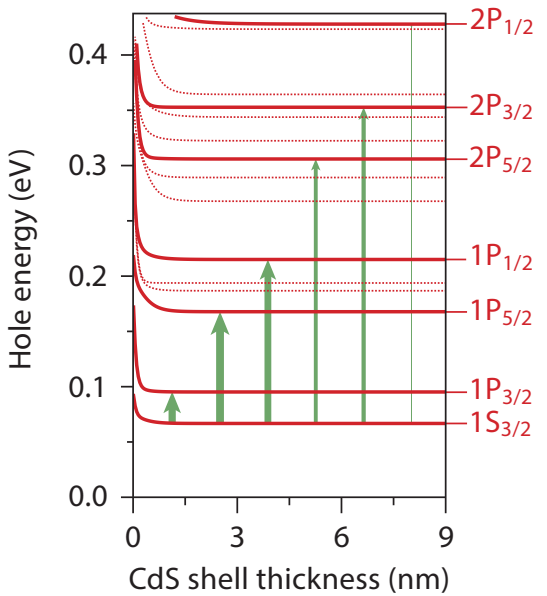

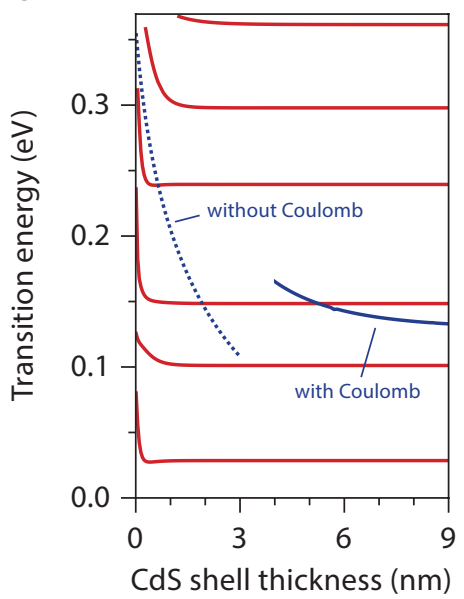

FIG. 6. (a) The radial components of $1 \mathrm{~S}_{\mathrm{e}}$ (blue) and $1 \mathrm{P}_{\mathrm{e}}$ (green) wavefunctions in CdSe/CdS core-shell QDs, for shell thicknesses of $1 \mathrm{~nm}, 5$ $\mathrm{nm}$ and $9 \mathrm{~nm}$. The confinement potential is due to the conduction band offset of $200 \mathrm{meV}$ and Coulomb attraction by the core-confined hole (red line in the bottom panel). In Supplementary Fig. S4 we show the wavefunction for a conduction band offset of $0 \mathrm{meV}$, and/or within the the strong-confinement approximation. (b) The energy level structure in CdSe/CdS core-shell QDs of hole states in the valence band, for a core diameter of $3.8 \mathrm{~nm}$ and as a function of shell thickness. Levels involved in $1 \mathrm{P}_{\mathrm{e}}-1 \mathrm{~S}_{\mathrm{e}}$ hot-electron cooling are indicated with solid lines, those not involved (because of selection rules) with dotted lines. The conduction band offset is set at $\Delta E_{\mathrm{cb}}=200 \mathrm{meV}$. The thickness of the upward arrows scales logarithmically with the Auger acceptor strength of the hole transition indicated (i.e. the Coulomb matrix element squared for the $1 \mathrm{P}_{\mathrm{e}} 1 \mathrm{~S}_{3 / 2} \rightarrow 1 \mathrm{~S}_{\mathrm{e}} n \mathrm{P}_{j}$ transition), calculated for a $3 \mathrm{~nm}$ thick shell. An increase of 0.5 point in thickness corresponds to a $10 \times$ stronger Auger coupling. (c) The energies of the transitions involved in hot-electron cooling, for a core diameter of $3.8 \mathrm{~nm}$ and as a function of shell thickness. Red lines are the hole transitions. The blue solid line is the $1 \mathrm{P}_{\mathrm{e}}-1 \mathrm{~S}_{\mathrm{e}}$ energy for $\Delta E_{\mathrm{cb}}=200$ meV and with the adiabatic Coulomb potential generated by a ground-state hole, while the blue dotted line represents the $1 \mathrm{P}_{\mathrm{e}}-1 \mathrm{~S}_{\mathrm{e}}$ energy calculated for the independent-particle electron states.

strongly phonon assisted, one might wonder if electron-hole Auger coupling is a dominant step in the relaxation process. Unfortunately, since in addition to the conduction band offset (which sets the $1 \mathrm{P}_{\mathrm{e}}-1 \mathrm{~S}_{\mathrm{e}}$ transition energy) the strength of phonon coupling is not precisely known, a quantitative theoretical prediction of the cooling pathways and rates is difficult.

If we follow the predominant assumption that hot-electron cooling is due to (phonon-assisted) Auger coupling with the hole, the independence of the CdS shell thickness (Fig. 5) can be qualitatively explained by speculating that there are several effects which can compensate each other. On the one hand, with increasing shell thickness the 1P-electron delocalizes in the CdS shell because core-confinement (due to the conduction band offset and Coulomb attraction by the hole) is weak in $\mathrm{CdSe} / \mathrm{CdS}$ (Fig. 6a). This effect would tend to reduce the electron-hole Auger coupling with the growth of a CdS shell. We propose two effects that could counteract this reduction. Firstly, the $1 \mathrm{P}_{\mathrm{e}}-1 \mathrm{~S}_{\mathrm{e}}$ energy gap becomes smaller with increasing $\mathrm{CdS}$ shell thickness (Fig. 6c). Since energy matching is needed of the $1 \mathrm{P}_{\mathrm{e}} \rightarrow 1 \mathrm{~S}_{\mathrm{e}}$ hot-electron transition in the conduction band to a transition in the valence band, a reduction of the $1 \mathrm{P}_{\mathrm{e}}-1 \mathrm{~S}_{\mathrm{e}}$ energy gap shifts the final hole state required to lower energy. We calculated the first-order Auger cooling matrix elements for the $1 \mathrm{P}_{\mathrm{e}} 1 \mathrm{~S}_{3 / 2} \rightarrow 1 \mathrm{~S}_{\mathrm{e}} n \mathrm{P}_{j}$ transitions, and found that the lower-energy hole transitions are stronger Auger acceptors than those at higher energy (see arrows in Fig. 6b). Consequently, the shift of the resonance condition with thicker shells in favor of the strong low-energy hole transitions could counteract the effect of electron delocalization. A second potentially compensating effect is that the exciton wavefunction for thick-shell QDs is more polarized than for thin-shell QDs because of increased spatial separation of electron and hole [55]. The coupling strength of the exciton to optical phonons should therefore increase, thus counteracting the effect of a smaller electron-hole overlap for phonon-assisted Auger coupling in thick-shell QDs. From our experimental result we can conclude that, under assumption of the Auger cooling mechanism, the effects of P-electron delocalization are roughly compensated by counteracting effects, leading to a seeming independence of the hot-electron cooling rate on shell thickness.

\section{CONCLUSION}

To summarize, we have examined the dynamics of biexciton Auger recombination and hot-electron cooling in $\mathrm{CdSe} / \mathrm{CdS}$ core-shell quantum dots having $3.8 \mathrm{~nm}$ diameter core with thin (1-2 monolayers) or thick (18 monolayers) shells. While Auger recombination of biexcitons is strongly suppressed in thick-shell quantum dots compared to thin shells, we have found that the intraband hot-electron cooling is nearly unaffected. We have described the suppression of biexciton Auger recombination theoretically, attributing it to a decrease of the electron-hole overlap due to electron delocalization with in- 
creasing shell thickness. Hot-electron cooling is generally believed to be driven by a mechanism similar to that of Auger recombination, and would therefore be expected to show a similar slow-down upon shell growth. To explain our observation of a nearly constant cooling rate, we propose that, within the Auger cooling scheme, the slow-down due to electron delocalization could be compensated by other effects. These could be due to the increased strength of the lower-energy hole transitions, which are gradually activated with increasing shell thickness, or due to enhanced coupling to optical phonons as the exciton wavefunction polarizes more with increasing shell thickness. Our calculations have indicated large energy gaps in the hole energy spectrum, independent of shell thickness (at least for $3.8 \mathrm{~nm}$ core diameter and smaller). This suggests that (for all but a few specific core-shell geometries) the Auger coupling of the hot-electron with the valence band hole must be strongly phonon-assisted, or accompanied by alternative cooling pathways. Our results contribute to the search for new methods to control nonradiative processes in colloidal quantum dots. Importantly, elimination of all nonradiative Auger processes is not always desired for applications. For example, for low-theshold lasing biexciton Auger decay should be inhibited while fast hot-electron cooling is a desired effect to maintain population inversion. Our work highlights the possibility of such independent control over different Auger processes.

\section{METHODS}

\section{Quantum dot synthesis}

Chemicals used. Cadmium acetate $\left(\mathrm{Cd}(\mathrm{Ac})_{2}\right.$, SigmaAldrich, 99\%), oleic acid (OA, Sigma-Aldrich, 90\%), octadecene (ODE, Sigma-Aldrich, 90\%), octadecyl amine (ODA, Sigma-Aldrich, 90\%) selenium (Strem Chemicals, 99.99\%), sulfur (Alfa Aesar, 99\%), trioctylphosphine (TOP, Sigma-Aldrich, 90\%), trioctylphosphine oxide (TOPO, Sigma-Aldrich, 99\%), acetone (Merck), hexane (SigmaAldrich, anhydrous, 99.8\%), methanol (Sigma-Aldrich, anhydrous, 99.8\%), 1-butanol (Sigma-Aldrich, anhydrous, 99.8\%), toluene (Sigma-Aldrich, anhydrous, 99.8\%).

Precursor preparation. Cadmium precursor I $[0.1 \mathrm{M}$ $\left.\mathrm{Cd}(\mathrm{OA})_{2}\right]$ was prepared by mixing OA (3.68 g), ODE (25.92 $\mathrm{g})$ and $\mathrm{Cd}(\mathrm{Ac})_{2}(0.64 \mathrm{~g})$, and heating to $120^{\circ} \mathrm{C}$ under vacuum for $2 \mathrm{~h}$. Cadmium precursor II [0.1 M Cd(OA)2] was prepared by dissolving $\mathrm{Cd}(\mathrm{Ac})_{2}(1.10 \mathrm{~g})$ in OA $(10.83 \mathrm{~g})$ and ODE (43.20 mL), and heating to $120^{\circ} \mathrm{C}$ under vacuum for 2 h. Selenium precursor was prepared by dissolving elemental selenium $(4.25 \mathrm{~g})$ in TOP $(22.5 \mathrm{~g})$ at $50^{\circ} \mathrm{C}$, followed by the addition of ODE (35.7 g). Sulfur precursor solution $(0.1 \mathrm{M})$ was prepared by dissolving sulfur $(0.032 \mathrm{~g})$ in ODE $(10 \mathrm{~mL})$ at $180^{\circ} \mathrm{C}$.

Synthesis of CdSe QD seeds. CdSe QD seeds were synthesized in a $50 \mathrm{~mL}$ three-neck flask using a Schlenk-line. TOPO (1.11 g), ODA (3.20 g) and Cd precursor I (4.9 g) were mixed, and heated to $295^{\circ} \mathrm{C}$. Se precursor $(5.2 \mathrm{~g})$ was then injected. The mixture was cooled down after $10 \mathrm{~min}$. The particles were diluted by adding 1 equivalent of hexane. The QDs were washed by adding 2 equivalents of methanol, collecting the upper hexane layer (colored), and then precipitating the QDs with 1 equivalent of acetone. Finally, the QDs were dissolved in toluene.

Growth of a CdS shell. The CdSe seeds $\left(10^{-7} \mathrm{M}\right.$ of QDs with $3.8 \mathrm{~nm}$ diameter in toluene), ODE (5.0 g) and ODA (1.5 g) were mixed and heated to $150^{\circ} \mathrm{C}$ for $1 \mathrm{~h}$ to remove all toluene. The reaction temperature was then increased to $240^{\circ} \mathrm{C}$. The shell was grown layer-by-layer under $\mathrm{N}_{2}$ by injecting a precursor solution (sufficient to form the next shell on all QDs) every $30 \mathrm{~min}$, alternating cation and anion precursors. The reaction solution was kept at $240^{\circ} \mathrm{C}$ for $1 \mathrm{~h}$, then allowed to cool down to room temperature, and diluted with 1 equivalent of hexane. The QDs were washed by adding 2 equivalents of methanol, collecting the upper hexane layer (colored), and then precipitating the QDs with 1 equivalent of acetone. Finally, the QDs were dissolved in toluene.

\section{Pump-push-probe transient absorption}

A film of QDs is deposited on an IR-transparent $\mathrm{CaF}_{2}$ substrate from a concentrated solution in toluene, and dried. For ultrafast experiments [58], the output of a regenerative $1 \mathrm{kHz}$ Ti:Sapphire amplifier system (Coherent, Legend Elite Duo, $800 \mathrm{~nm}, 40 \mathrm{fs}$ pulse duration, $7 \mathrm{~mJ}$ per pulse) was split into three parts. One part was frequency doubled in a BBO crystal to generate $400-n m 50-f$ s visible pump pulses. The second part was used to generate mid-IR push pulses by pumping a commercial parametric amplifier with a difference frequency generation (DFG) stage (HE TOPAS, $80 \mathrm{fs}, 0.22 \mathrm{eV}$ photons). The rest was used to pump a 3-stage home-built optical parametric amplifier followed by a DFG stage to provide independently tunable $70 \mathrm{fs}$ IR $(0.21 \mathrm{eV})$ probe and reference pulses. Slight detuning of push and probe frequencies was done to minimize background of scattered push light in the detection path.

All beams were focused on the sample using a $20 \mathrm{~cm}$ concave parabolic mirror. The time delays of pump and probe were controlled using mechanical delay stages. In the pump-probe measurement a $500 \mathrm{~Hz}$ mechanical modulator was set in the pump beam path and for pump-push-probe measurements the modulator was moved to the push beam path. All beams had the same (p) polarization. The probe and reference IR beams passed through the sample and were spectrally dispersed and detected by a nitrogen-cooled mercury cadmium telluride detector array. The measurements were performed under $\mathrm{N}_{2}$ flow to avoid water vapor absorption of IR light and sample degradation. 


\section{Single-QD spectroscopy}

For single-QD measurements the QDs were spin-coated on a glass cover slip from a dilute dispersion in toluene, and covered with a layer of PMMA. They were excited with $10 \mathrm{ps,}$ $10 \mathrm{MHz}, 532 \mathrm{~nm}$ laser pulses through an $100 \times$ oil immersion objective with NA 1.4. From the absorption cross-section of the QDs ( $\sigma_{\text {abs }} \approx 10^{-15} \mathrm{~cm}^{2}$ at $532 \mathrm{~nm}$ [59]) we estimate that the laser power of $200 \mathrm{nW}$ focused to a diffraction-limited spot correspond to the generation of $\langle N\rangle \approx 0.1$ excitons per laser pulse.

\section{Theoretical model}

The calculations of the biexciton Auger recombination rate in $\mathrm{CdSe} / \mathrm{CdS}$ core-shell QDs were performed within an 8band $\mathbf{k} \cdot \mathbf{p}$ model using a scheme similar to the one described in Ref. 35 where core-only CdSe QDs were addressed. The modification in the model presented here is the addition of a CdS shell layer, which produces QDs having two heterointerfaces: one between the CdSe core and the CdS shell, and another between the $\mathrm{CdS}$ shell and the environment. The wave functions of the electrons and holes are written in the form $\boldsymbol{\Psi}=\left(\Psi_{\mathrm{c}}, \boldsymbol{\Psi}_{\mathrm{v}}\right)$, where $\Psi_{\mathrm{c}}$ is the two-component conduction band spinor envelope function, and $\Psi_{\mathrm{v}}=\left(\Psi_{x}, \Psi_{y}, \Psi_{z}\right)$ is the valence band spinor envelope vector [60]. The procedure of finding the wave functions is the same as in Ref. 35, except for the finite potential barriers for holes assumed here, and the modified boundary conditions. In finding the electron wave functions of both ground and excited states, the boundary conditions imposed at each of the interfaces are those given in Eq. (3) in Ref. 35 with the matrix $\hat{T}$ set to unity and $\lambda=0$.

For ground state (bound) hole wave functions, the boundary conditions at the core-shell interface are the continuity of each row of the follow vector:

$$
\left(\begin{array}{c}
\boldsymbol{\tau} \cdot \boldsymbol{\Psi}_{\mathrm{v}} \\
\boldsymbol{\tau} \times \boldsymbol{\Psi}_{\mathrm{v}} \\
\left(\frac{E_{\mathrm{p}}}{E_{\mathrm{c}}-E}+\beta_{\ell}-\beta_{h}\right)\left(\nabla \cdot \boldsymbol{\Psi}_{\mathrm{v}}\right)+\beta_{h} \frac{\partial}{\partial r}\left(\boldsymbol{\tau} \cdot \boldsymbol{\Psi}_{\mathrm{v}}\right) \\
\beta_{h} \frac{\partial}{\partial r}\left(\boldsymbol{\tau} \times \boldsymbol{\Psi}_{\mathrm{v}}\right)
\end{array}\right)
$$

where $\tau$ is the radial unit vector, $E_{\mathrm{p}}=2 m_{0} P^{2}$ with $m_{0}$ the free electron mass and $P$ the Kane parameter, $E_{c}$ is the conduction band edge energy, $E$ is the energy of the hole state, $\beta_{\ell}=\gamma_{1}+4 \gamma$ and $\beta_{h}=\gamma_{1}-2 \gamma$, where $\gamma, \gamma_{1}$ are modified Luttinger parameters. To describe the excited (unbound) hole states, we assume the flat-band approximation in which $\beta_{h}=0$. This simplifies the excited hole boundary conditions at the core-shell interface, which reduce to the continuity of:

$$
\left(\begin{array}{c}
\boldsymbol{\tau} \cdot \boldsymbol{\Psi}_{\mathrm{v}} \\
\left(\frac{E_{\mathrm{p}}}{E_{\mathrm{c}}-E}+\beta_{\ell}\right)\left(\nabla \cdot \boldsymbol{\Psi}_{\mathrm{v}}\right)
\end{array}\right)
$$

Outside the QD, the hole, just like the electron, is described by a free-particle two-component spinor wave function. It then follows that the boundary conditions for the ground state hole at the shell-environment interface are the equality of the first and third rows in Eq. (1) here with the right-handside of Eq. (3) in Ref. 35, with an additional condition of $\left(\boldsymbol{\tau} \times \boldsymbol{\Psi}_{\mathrm{v}}\right)=0$ at this interface. The boundary conditions for the excited holes at the shell-environment interface are the equality of Eq. (2) here with the right-hand-side of Eq. (3) in Ref. [35]. Once the electron and hole wave functions and energies are found, the calculation of the Coulomb integrals that enter the matrix elements of Auger recombination and Auger cooling is the same as in Ref. [35].

\section{ACKNOWLEDGEMENTS}

This work is part of the research program of the "Stichting voor Fundamenteel Onderzoek der Materie (FOM)", which is financially supported by the "Nederlandse Organisatie voor Wetenschappelijk Onderzoek (NWO)". A.L.E. acknowledges the financial support of the Office of Naval Research (ONR) through the Naval Research Laboratory Basic Research Program.

[1] Nirmal, M., Dabbousi, B. O., Bawendi, M. G., Macklin, J. J., Trautman, J. K., Harris, T. D. \& Brus, L. E. Fluorescence intermittency in single cadmium selenide nanocrystals, Nature $\mathbf{3 8 3}$, 802-804 (1996)

[2] Efros, Al. L. \& Rosen, M. Random telegraph signal in the photoluminescence intensity of a single quantum dot, Phys. Rev. Lett. 78, 6, 1110-1113 (1997)

[3] Galland, C. et al. Two types of luminescence blinking revealed by spectroelectrochemistry of single quantum dots, Nature $\mathbf{4 7 9}$, 203-207 (2011)

[4] Bae, W. K. et al. Controlling the influence of Auger recombination on the performance of quantum-dot light-emitting diodes, Nat. Commun. 4, 2661 (2013)

[5] Jang, E. et al. White-light-emitting diodes with quantum dot color converters for display backlights, Adv. Mater. 22 30763080 (2010)

[6] Resch-Genger, U., Grabolle, M., Cavaliere-Jaricot, S., Nitschke, R. \& Nann, T. Quantum dots versus organic dyes as fluorescent labels, Nat. Methods 5, 763-775 (2008)

[7] Mikhailovsky, A. A., Malko, A. V., Hollingsworth, J. A., Bawendi, M. G. \& Klimov, V. I. Multiparticle interactions and stimulated emission in chemically synthesized quantum dots, Appl. Phys. Lett. 80, 2380-2382 (2002)

[8] Dang, C. et al. Red, green and blue lasing enabled by singleexciton gain in colloidal quantum dot films, Nat. Nanotechnol. 7, 335-339 (2012)

[9] Grim, J. G. et al. Continuous-wave biexciton lasing at room temperature using solution-processed quantum wells, Nat. Nanotechnol. 9, 891-895 (2014)

[10] Efros, Al. L., Kharchenko, V. A. \& Rosen, M. Breaking the phonon bottleneck in nanometer quantum dots: role of Augerlike processes, Solid State Commun. 93, 281-284 (1995) 
[11] Hendry, E., Koeberg, M., Wang, F., Zhang, H., De Mello Donegá, C., Vanmaekelbergh, D. \& Bonn, M. Direct observation of electron-to-hole energy transfer in CdSe quantum dots, Phys. Rev. Lett. 96, 057408 (2006)

[12] Deng, Z., Jeong, K. S. \& Guyot-Sionnest, P. Colloidal quantum dots intraband photodetectors, ACS Nano 8, 11707-11714 (2014)

[13] Tisdale, W. A. et al. Hot-electron transfer from semiconductor nanocrystals, Science 328 1543-1547 (2010)

[14] Klimov, V. I. Spectral and dynamical properties of multiexcitons in semiconductor nanocrystals, Annu. Rev. Phys. Chem. 58, 635-673 (2007)

[15] Cragg, G. E. \& Efros, Al. L. Suppression of Auger processes in confined structures, Nano Lett. 10, 313-317 (2010)

[16] Mahler, B. et al. Towards non-blinking colloidal quantum dots, Nat. Mater. 7, 659-664 (2008)

[17] Hollingsworth, J. A. Heterostructuring nanocrystal quantum dots toward intentional suppression of blinking and Auger recombination, Chem. Mater. 25, 1318-1331 (2013)

[18] García-Santamaría, F. et al. Breakdown of volume scaling in Auger recombination in $\mathrm{CdSe} / \mathrm{CdS}$ heteronanocrystals: the role of the core-shell interface, Nano Lett. 11, 687-693 (2011)

[19] Bae, W. K. et al. Controlled alloying of the core-shell interface in $\mathrm{CdSe} / \mathrm{CdS}$ quantum dots for suppression of Auger recombination, ACS Nano 7, 3411-3419 (2013)

[20] Nasilowski, M., Spinicelli, P., Patriarche, G. \& Dubertret, B. Gradient $\mathrm{CdSe} / \mathrm{CdS}$ quantum dots with room temperature biexciton unity quantum yield, Nano Lett. 15, 3953-3958 (2015)

[21] Vaxenburg, R., Lifshitz, E. \& Efros, Al. L. Suppression of Auger-stimulated efficiency droop in nitride-based light emitting diodes, Appl. Phys. Lett. 102, 031120 (2013)

[22] Dennis, A. M., Mangum, B. D., Piryatinski, A., Park, Y.-S., Hannah, D. C., Casson, J. L., Williams, D. J., Schaller, R. D., Htoon, H. \& Hollingsworth, J. A. Suppressed blinking and Auger recombination in near-infrared type-II InP/CdS nanocrystal quantum dots, Nano Lett. 12, 5545-5551 (2012)

[23] Guyot-Sionnest, P., Shim, M., Matranga, C. \& Hines, M. Intraband relaxation in CdSe quantum dots, Phys. Rev. B 60, R2181R2184 (1999)

[24] Pandey, A. \& Guyot-Sionnest, P. Slow electron cooling in colloidal quantum dots, Science 322, 929-932 (2008)

[25] Klimov, V. I. \& McBranch, D. W. Femtosecond 1P-to-1S electron relaxation in strongly confined semiconductor nanocrystals, Phys. Rev. Lett. 80, 4028-4031 (1998)

[26] Klimov, V. I., McBranch, D. W., Leatherdale, C. A. \& Bawendi, M. G. Electron and hole relaxation pathways in semiconductor quantum dots, Phys. Rev. B 60, 13740-13749 (1999)

[27] Sippel, P., Albrecht, W., Mitoraj, D., Eichberger, R., Hannappel, T. \& Vanmaekelbergh, D. Two-photon photoemission study of competing Auger and surface-mediated relaxation of hot electrons in CdSe quantum dot solids, Nano Lett. 13, 1655-1661 (2013)

[28] Klimov, V. I., Mikhailovsky, A. A., McBranch, D. W., Leatherdale, C. A. \& Bawendi, M. G. Quantization of multiparticle Auger rates in semiconductor quantum dots, Science $\mathbf{2 8 7}$ 1011-1013 (2000)

[29] Cohn, A. W., Rinehart, J. D., Schimpf, A. M. Weaver, A. L. \& Gamelin, D. R. Size dependence of negative trion Auger recombination in photodoped CdSe nanocrystals, Nano Lett. 14, 353-358 (2014)

[30] Park, Y.-S. et al. Near-unity quantum yields of biexciton emission from $\mathrm{CdSe}-\mathrm{CdS}$ nanocrystals measured using singleparticle spectroscopy, Phys. Rev. Lett. 106, 187401 (2011)
[31] Zhao, J., Chen, O., Strasfeld, D. B. \& Bawendi, M. G. Biexciton quantum yield heterogeneities in single $\mathrm{CdSe}(\mathrm{CdS})$ core (shell) nanocrystals and its correlation to exciton blinking, Nano Lett. 12, 4477-4483 (2012)

[32] Galland, C., Ghosh, Y., Steinbrück, A. Hollingsworth, J. A., Htoon, H. \& Klimov, V. I. Lifetime blinking in nonblinking nanocrystal quantum dots, Nat. Commun. 3, 908 (2012)

[33] Park, Y.-S., Bae, W. K., Padilha, L. A., Pietryga, J. M. \& Klimov, V. I. Effect of the core/shell interface on Auger recombination evaluated by single-quantum-dot spectroscopy, Nano Lett. 14, 396-402 (2014)

[34] Chepic, D. I. et al. Auger ionization of semiconductor quantum drops in a glass matrix, J. Lumin. 47, 113-127 (1990)

[35] Vaxenburg, R., Rodina, A., Shabaev, A., Lifshitz, E. \& Efros, Al. L. Nonradiative Auger recombination in semiconductor nanocrystals, Nano Lett. 15, 2092-2098 (2015)

[36] Kilina, S. V., Kilin, D. S. \& Prezhdo, O. V. Breaking the phonon bottleneck in PbSe and CdSe quantum dots: time-domain density functional theory of charge carrier relaxation, ACS Nano 3 , 93-99 (2009)

[37] Cooney, R. R., Sewall, S. L., Anderson, K. E. H., Dias, E. A. \& Kambhampati, P., Breaking the phonon bottleneck for holes in semiconductor quantum dots, Phys. Rev. Lett. 98, 177403 (2007)

[38] Park, Y.-S., Bae, W. K., Pietryga, J. M. \& Klimov, V. I. Auger recombination of biexcitons and negative and positive trions in individual quantum dots, ACS Nano 8, 7288-7296 (2014)

[39] Norris, D. J. \& Bawendi, M. G., Measurement and assignment of the size-dependent optical spectrum in CdSe quantum dots, Phys. Rev.B 53, 16338-16346 (1996)

[40] Guyot-Sionnest, P. \& Hines, M. A. Intraband transitions in semiconductor nanocrystals, Appl. Phys. Lett. 72, 686-688 (1998)

[41] Achermann, M., Hollingsworth, J. A. \& Klimov, V. I. Multiexcitons confined within a subexcitonic volume: spectroscopic and dynamical signatures of neutral and charged biexcitons in ultrasmall semiconductor nanocrystals, Phys. Rev. B 68, 245302 (2003)

[42] Pandey, A. \& Guyot-Sionnest, P. Multicarrier recombination in colloidal quantum dots, J. Chem. Phys. 127, 111104 (2007)

[43] Nair, G., Zhao, J. \& Bawendi, M. G. Biexciton quantum yield of single semiconductor nanocrystals from photon statistics, Nano Lett. 11, 1136-1140 (2011)

[44] Deutsch, Z., Schwartz, O., Tenne, R., Popovitz-Biro, R. \& Oron, D. Two-color antibunching from band-gap engineered colloidal semiconductor nanocrystals, Nano Lett. 12, 29482952 (2012)

[45] Mangum, B. D., Ghosh, Y., Hollingsworth, J. A. \& Htoon, H. Disentangling the effects of clustering and multiexciton emission in second-order photon correlation experiments, Opt. Express 21, 7419-7426 (2013)

[46] De Mello Donegá, C. Synthesis and properties of colloidal heteronanocrystals, Chem. Soc. Rev. 40, 1512-1546 (2011)

[47] Müller, J. et al. Wave function engineering in elongated semiconductor nanocrystals with heterogeneous carrier confinement, Nano Lett. 5, 2044-2049 (2005)

[48] Steiner, D. et al. Determination of band offsets in heterostructured colloidal nanorods using scanning tunneling spectroscopy, Nano Lett. 8, 2954-2958 (2008)

[49] Javaux, C. et al. Thermal activation of non-radiative Auger recombination in charged colloidal nanocrystals, Nat. Nanotechnol. 8, 206-212 (2013)

[50] Ekimov, A. I. et al. Absorption and intensity-dependent photoluminescence measurements on CdSe quantum dots: assignment of the first electronic transitions, J. Opt. Soc. Am. B 10, 100-107 (1993) 
[51] Efros, Al. L. \& Rosen, M. Quantum size level structure of narrow-gap semiconductor nanocrystals: effect of band coupling, Phys. Rev. B 58, 7120-7135 (1998)

[52] Li, J. J., Wang, A., Guo, W., Keay, J. C., Mishima, T. D., Johnson, M. B. \& Peng, X. Large-scale synthesis of nearly monodisperse $\mathrm{CdSe} / \mathrm{CdS}$ core/shell nanocrystals using air-stable reagents via successive ion layer adsorption and reaction, J. Am. Chem. Soc. 125, 12567-12575 (2003)

[53] Roussignol, P., Kull, M., Ricard, D., De Rougemont, F., Frey, R. $\&$ Flytzanis, C. Timeresolved direct observation of Auger recombination in semiconductor-doped glasses, Appl. Phys. Lett. 51, 1882-1884 (1987)

[54] Shabaev, A., Rodina, A. V. \& Efros, Al. L. Fine structure of the band edge excitons and trions in $\mathrm{CdSe} / \mathrm{CdS}$ core/shell nanocrystals, Phys. Rev. B 86, 205311 (2012)

[55] Schmitt-Rink, S., Miller, D. A. B. \& Chemla, D. S. Theory of the linear and nonlinear optical properties of semiconductor microcrystallites, Phys. Rev. B 35, 8113-8125 (1987)

[56] Bakulin, A. A., Rao, A., Pavelyev, V. G., Van Loosdrecht, P. H. M., Pshenichnikov, M. S., Niedzialek, D., Cornil, J., Beljonne, D. \& Friend, R. H. The role of driving energy and delocalized states for charge separation in organic semiconductors, Science 335, 1340-1344 (2012)

[57] Smith, A. M., Mohs, A. M. \& Nie, S. Tuning the optical and electronic properties of colloidal nanocrystals by lattice strain, Nat. Nanotechnol. 4, 56-63 (2009)

[58] Bakulin, A. A., Neutzner, S., Bakker, H. J., Ottaviani, L., Barakel, D. \& Chen, Z. Charge trapping dynamics in PbS colloidal quantum dot photovoltaic devices. ACS Nano 7, 87718779 (2013)

[59] Leatherdale, C. A., Woo, W.-K., Mikulec, F. V. \& Bawendi, M. G. On the absorption cross section of CdSe nanocrystal quantum dots, J. Phys. Chem. B 106, 7619-7622 (2002)

[60] Rodina, A. V., Efros, Al. L. \& Alekseev, A. Yu. Effect of the surface on the electron quantum size levels and electron $g$-factor in spherical semiconductor nanocrystals, Phys. Rev. B 67, 155312 (2003) 